medication. (Zametkin AJ, Rapoport JL. Neurobiology of ADHD. J Am Acad Child Adolesc Psychiatry 1987;26:676-686).

\title{
PERIPHERAL NERVOUS SYSTEM DISORDERS
}

\section{EARLY- AND LATE-ONSET INHERITED ERYTHROMELALGIA}

A genotype-phenotype relationship at the clinical, cellular and molecular levels is shown in a case of erythromelalgia of relatively late onset, in a study at Yale University School of Medicine, and centers in China. The patient, a male age 17 years, began experiencing excruciating pain, warmth and redness in both feet and lower legs at age 14 years. Blood was analyzed for mutations in SCN9A, gain-of-function sodium channel mutations that are preferentially expressed within dorsal root ganglia (DRG) and sympathetic ganglion neurons. The effect of the Q10R mutation on firing of DRG neurons was investigated by current-clamp recording. The hyperexcitability of the DRG neuron induced by Q10R mutation in this adolescent patient was smaller than the change produced by I848T, an early-onset erythromelalgia mutation. (Han C, Dib-Hajj SD, Lin Z et al. Early- and lateonset inherited erythromelalgia: genotype-phenotype correlation. Brain July 2009;132:17111722). (Respond: Stephen G Waxman, MD PhD, Department of Neurology, LCI 707, Yale University School of Medicine, 333 Cedar Street, New Haven, CT 06520. E-mail: Stephen.Waxman@yale.edu).

COMMENT. Inherited erythromelalgia (erythermalgia) (IEM) is an autosomal dominant disorder characterized by severe burning pain and erythema of the extremities triggered by warmth. IEM is linked to gain-of-function mutations in SCN9A, the gene encoding $\mathrm{Na}, 1.7$, a voltage-gated sodium channel that is preferentially expressed in dorsal root ganglion neurons, particularly nociceptors, and sympathetic ganglion neurons. The resultant nociceptor hyperexcitability causes pain in the extremities. Almost all cases reported are linked to families with onset in early childhood (infancy to 6 years of age). The above case report uncovers a new mutation in $\mathrm{Na}, 1.7, \mathrm{Q} 10 \mathrm{R}$, from a patient with onset in the second decade of life. Mutations that produce smaller effects on sodium channel activation are associated with a smaller degree of DRG neuron excitability and later onset of clinical signs.

Carbamazepine-responsive erythromelalgia and $\mathrm{Na}_{\mathrm{v}} 1.7$ mutation. The above team of investigators publish a second article on erythromelalgia (Fischer TZ et al. Ann Neurol July 2009;65:733-741), reporting a novel $\mathrm{Na}_{\mathrm{v}} 1.7$ mutation (V400M) in a threegeneration Canadian family with pain relieved by carbamazepine (CBZ). $\mathrm{Na}_{\mathrm{v}} 1.7$ sodium channels are preferentially expressed within nociceptor ganglia and sympathetic neurons that are involved in the inflammatory and neuropathic pain of inherited erythromelalgia. CBZ has a normalizing effect on mutant $\mathrm{Na}_{\mathrm{v}} 1.7$ channels in this Canadian kindred, preventing the hyperexcitability of dorsal root ganglia in erythromelalgia. 